\title{
Investigation of Integrated Behavior Therapy for Selective Mutism: A Replicated Single-Case Study Design
}

\author{
Allison K. Siroky ${ }^{1}$, John S. Carlson ${ }^{1} \&$ Aimee Kotrba ${ }^{2}$ \\ ${ }^{1}$ Department of Counseling, Educational Psychology, and Special Education, Michigan State University, East \\ Lansing, MI, USA \\ ${ }^{2}$ Thriving Minds Behavioral Health Center, Brighton, MI, USA \\ Correspondence: Allison K. Siroky, Department of Counseling, Educational Psychology, and Special Education, \\ Michigan State University, East Lansing, MI, USA. E-mail: sirokyal@msu.edu
}

Received: February 7, 2017

Accepted: March 12, 2017

Online Published: April 6, 2017

doi:10.5539/ijps.v9n2p82

URL: http://doi.org/10.5539/ijps.v9n2p82

\begin{abstract}
Selective Mutism (SM) is a rare but potentially debilitating disorder characterized by a lack of speech in certain settings where speaking is expected. This study examined the effectiveness of a shortened version (12 sessions over 18 weeks) of Integrated Behavior Therapy for Selective Mutism (IBTSM; Bergman, 2013) in increasing speech and relieving anxiety for two four-year-old males with SM via a replicated single-case design. Treatment effectiveness, integrity, and acceptability were measured at baseline, throughout treatment, and at a three-month follow-up. Treatment integrity was excellent for both cases. SM severity ratings decreased from baseline to end-of-treatment, and again at follow-up, for each case. Verbal communication increased at end-of-treatment and follow-up, and significant decreases in social anxiety were seen across both cases by the three-month follow-up. Parents rated the shortened IBTSM as highly acceptable, effective, and efficient. Future studies should explore the effectiveness of varying lengths of IBTSM.
\end{abstract}

Keywords: behavioral therapy, manualized treatment, selective mutism, social anxiety

\section{Introduction}

Selective Mutism (SM) is a rare but potentially debilitating anxiety disorder involving a persistent failure to speak in certain situations despite normal speech in others. Initial symptoms are typically seen before age five and tend to coincide with the transition to formal schooling (American Psychiatric Association [APA], 2013). The most recent edition of the Diagnostic and Statistical Manual of Mental Disorders (DSM-5; APA, 2013) classifies SM as an anxiety disorder, attesting to the more widely understood theory that children with SM fail to speak due to some underlying anxiety associated with speech or social situations. SM can drastically hinder a child's school performance and social competence at a critical time in their development (Steinhausen, Wachter, Laimböck, \& Metzke, 2006). Thus, knowledge about SM and its treatment is imperative for practitioners in both clinical and school settings.

Muris and Ollendick (2015) note the popularity and effectiveness of behavioral interventions for the treatment of SM, though few programs are meant specifically for children with SM. Integrated Behavior Therapy for Selective Mutism (IBTSM; Bergman, 2013) is a 20-session weekly treatment, which uses common behavioral strategies to elicit speech across settings. The treatment developers examined the efficacy of IBTSM for 21 children diagnosed with SM via a randomized controlled trial (Bergman, Gonzalez, Piacentini, \& Keller, 2013). Children receiving IBTSM $(n=12)$ showed significant increases in speech and decreases in social anxiety compared to the waitlist group. Further, $67 \%$ of the treatment group did not meet criteria for SM at the end of treatment. Significant improvements in speech and social anxiety were also seen at the midpoint assessment (12 weeks). As such, Bergman and colleagues (2013) indicated that a shorter version of IBTSM may be effective for young children. No investigations to date have explored the potential benefit of a modified treatment length.

\subsection{Purpose and Hypotheses of Present Study}

The purpose of the present study was to explore the potential effectiveness of a shortened, 12-session version of the IBTSM program in reducing SM symptoms for two young children. The replicated single-case AB design allowed for close monitoring of incremental changes in speech and social anxiety over time. Three hypotheses 
were proposed: (1) The study therapist will implement the 12-session version of IBTSM with adequate treatment integrity for both cases, (2) A shortened version of IBTSM will successfully relieve speech avoidance and social anxiety for these two cases as indicated by noticeable reductions in parent-rated symptoms, and (3) Parents of both cases will perceive the shortened version of IBTSM as acceptable, effective, and sufficient with regard to time required.

\section{Method}

\subsection{Design}

This study used a replicated single-case design to assess the feasibility, effectiveness, and acceptability of IBTSM for two young children diagnosed with SM receiving treatment within a community-based clinic. Parents completed assessments of speech and social anxiety at baseline, midpoint, end-of-treatment, and follow-up, as well as weekly social anxiety ratings. Attempts to gather teacher data were undertaken but unsuccessful for both cases and thus not reported. No randomization or blinding was used in this replicated $\mathrm{AB}$ design.

\subsection{Participants}

The human subjects review board at the first authors' institution approved of this project prior to recruitment and data collection. Two families were recruited from a pediatric psychology clinic that specializes in SM. Children receiving any other form of treatment for SM symptoms were excluded from consideration. Children also needed to be enrolled in preschool due to the use of school-based exposure activities throughout IBTSM. Informed consent and assent were obtained prior to the first treatment session.

Participants were both four-year-old males attending their second year of preschool. Both children began treatment with moderate (Child 1, African-American) to severe (Child 2, Caucasian) SM. At baseline, parent-reported speaking behaviors indicated infrequent speech across settings for Child 1 (Table 1). Child 1 also met criteria for comorbid social phobia and separation anxiety disorder at baseline. Child 2 did not meet criteria for other disorders, though parent ratings suggest Child 2 presented with less frequent speech across settings, when compared to Child 1.

\subsection{Intervention}

A licensed psychologist with considerable experience in treating SM served as the study psychologist after receiving additional training with the IBTSM manual. IBTSM consists of 20 in-therapy sessions over 24 weeks, with additional behavioral tasks for the child to complete at home, at school, or in public settings (Bergman, 2013). The present study condensed this intervention to 1245 -minute sessions, though all key components of IBTSM (e.g., pre-treatment parent session, fear hierarchy, in-session exposures, out-of-session homework assignments) were retained. For both participants, 18 weeks lapsed between the first and the final session due to unanticipated cancellations (e.g., holidays, sickness, weather). Parents were involved in all treatment sessions.

The first two sessions of IBTSM focused on building rapport, establishing behavioral control (i.e., when prompted to speak, the child responds), and introducing key behavioral tools (e.g., contingency management, feelings "thermometer", fear hierarchy). The focus transitioned to repeated exposure therapy, guided by each child's "fear ladder". Sessions 3 through 5 emphasized in-session exposure activities to promote speech in new contexts and with new people. Exposure exercises became progressively more difficult as they extended to the classroom, playdates with peers, and spontaneous interactions in public. In the remaining weeks of treatment (Sessions 7-11), parents took on greater responsibility in facilitating exposures, home assignments, and the contingency management plan. The final session (Session 12) focused on celebrating the child's progress and giving parents resources to prevent relapse.

\subsection{Measures}

\subsubsection{Treatment Integrity}

Treatment integrity checklists were created for all 12 sessions using session outlines from the IBTSM manual (Bergman, 2013). The first author observed in-clinic sessions live or via videotape noting the number of items completed. At the end of the treatment, average percentages determined overall integrity. Three of Child 1's sessions (Sessions 9-11) could not be assessed because they took place outside of the clinic (i.e., in public) and were not recorded on video.

\subsubsection{Clinical Diagnoses}

The Anxiety Disorders Interview Schedule for Children, Parent Version (ADIS-P; Silverman \& Albano, 1996) informed diagnostic status at baseline, after the final treatment session, and again at the three-month follow-up. The ADIS-P is a comprehensive structured interview intended to evaluate a child's symptoms of anxiety and 
clinical severity. Parent responses result in a Clinical Severity Rating (CSR), which can range from zero to eight. As suggested by the developers, a CSR at or above four was considered to be within the clinical range suggesting that the child's presenting behaviors met diagnostic criteria and confirmed a clinical diagnosis (Silverman \& Albano, 1996). Silverman, Saavedra, and Pina (2001) report good to excellent reliability for diagnosing anxiety disorders listed in the DSM-IV $(\kappa=0.65-0.88)$.

\subsubsection{Speaking Behaviors}

The Selective Mutism Questionnaire (SMQ; Bergman, Keller, Piacentini, \& Bergman, 2008) was given to each child's parent at baseline and all subsequent assessment periods (i.e., midpoint, end-of-treatment, follow-up). The SMQ consists of 17 items related to the frequency of their child's speech in three domains: Home, School, and Other contexts. Total scores range from 0-51 with higher scores indicating increased frequency of speech. The IBTSM pilot study revealed a Cronbach's alpha of $\alpha=.76$ (Bergman et al., 2013).

\subsubsection{Social Anxiety}

Parents completed the Preschool Anxiety Scale-Parent Report, Social Anxiety Subscale (PAS-P; Spence, Rapee, McDonald, \& Ingram, 2001) each week to assess their child's level of social anxiety over time. The PAS-P consists of 28 items total, though only items from the social anxiety subscale (6 items) were administered. Higher scores represent greater severity of anxious symptoms. The six-item social anxiety subscale has moderate reliability ( $\alpha=.75$; Muris, Schmidt, \& Merckelbach, 2000).

In addition, the Social Anxiety Scale for Children-Revised, Parent Version (SASC-P; La Greca \& Stone, 1993) was administered to parents at baseline, midpoint, end-of-treatment, and follow-up sessions. The SASC-P is an 18-item questionnaire, adapted for parents to evaluate the severity of their child's social anxiety symptoms. Three subscales of the SASC-P label items into areas of fear of negative evaluation (FNE; 8 items), social avoidance and distress in new situations (SAD-New; 6 items), or general social avoidance and distress (SAD-G; 4 items). Bergman and colleagues (2013) reported good reliability $(\alpha=.87)$ for the parent form.

\subsubsection{Treatment Acceptability}

The Treatment Evaluation Questionnaire (TEQ; Kelley, Heffer, Gresham, \& Elliott, 1989) was given at the end of treatment and at the three-month follow-up to gather parent satisfaction ratings of IBTSM. The TEQ contains 21 items with three subscales pertaining to Acceptability (11 items), Effectiveness ( 8 items), and Time Required ( 2 items). Higher overall scores indicate higher acceptability. The TEQ was adapted from the Treatment Evaluation Inventory (Kazdin, 1980), which has strong internal consistency $(\alpha=.97)$.

\subsection{Analyses}

Data representing weekly parent ratings of social anxiety (i.e., PAS-P) were analyzed via visual analysis, with supplemental effect size calculations. Reliable Change Index (RCI; Jacobson \& Truax, 1991) scores determined the clinical significance of changes in speech and social anxiety. RCI scores were calculated by subtracting each child's end-of-treatment or follow-up score from their baseline score, and dividing this by the standard error of measurement for a given measure. Significant RCI scores $( \pm 1.96)$ were used to confirm changes in clinical diagnoses as demonstrated by responses on the ADIS-P from baseline to end-of-treatment, and at the three-month follow-up. We referred to previous studies with similar samples for SMQ and SASC-P reliability coefficients and standard deviations to calculate the standard error of measurement (Bergman et al., 2008; Bergman et al., 2013; Letamendi et al., 2008).

\section{Results}

\subsection{Treatment Integrity}

The participating psychologist maintained an average of $96.7 \%$ integrity throughout treatment for both cases despite slight modifications (e.g., reviewing strategies learned in earlier sessions, facilitating playdates) to meet the needs of each family. Findings were consistent with those of Bergman and colleagues (2013), who reported excellent integrity $(M=99.3 \%)$ and session quality $(M=9.79 / 10, S D=.51)$ for IBTSM in its original format.

\subsection{Speaking Behaviors}

Upon completing the final session, both children continued to meet criteria for SM based on ADIS-P scores; however, both cases saw a decrease in overall symptom severity over time as indicated by ADIS-P clinical severity ratings (CSRs; Table 1). By the three-month follow-up interview, Child 1 no longer met criteria for SM. Child 2 continued to meet criteria for SM even at the follow-up, though his mother reported a noticeable decrease in SM severity. Similarly, frequency of parent-reported speech via the SMQ increased over time for 
both children, with maintenance three months after treatment. Only Child 1 made clinically significant improvements in speech at the end-of-treatment $(R C I=2.44)$ and follow-up $(R C I=2.52)$ assessments.

Table 1. Parent ratings on dependent variables over time

\begin{tabular}{|c|c|c|c|c|c|c|c|c|}
\hline & \multicolumn{4}{|c|}{ Child 1} & \multicolumn{4}{|c|}{ Child 2} \\
\hline & Baseline & Midpoint & $\begin{array}{c}\text { End-of-treat } \\
\text { ment }\end{array}$ & Follow-up & Baseline & Midpoint & $\begin{array}{c}\text { End-of-treat } \\
\text { ment }\end{array}$ & Follow-up \\
\hline \multirow{2}{*}{\multicolumn{9}{|c|}{$\begin{array}{l}\text { ADIS-P, } \\
\text { Clinical Severity Rating }\end{array}$}} \\
\hline & & & & & & & & \\
\hline Selective Mutism & 6 & -- & 5 & $n / a$ & 7 & -- & 6 & 4 \\
\hline Separation Anxiety & 6 & -- & 4 & 4 & $n / a$ & -- & $n / a$ & $n / a$ \\
\hline Social Phobia & 7 & -- & 4 & 4 & $n / a$ & -- & $n / a$ & $n / a$ \\
\hline SMQ Total Score & 17 & 22 & $26^{*}$ & $30^{*}$ & 5 & 8 & 11 & 12 \\
\hline School & 4 & 8 & 10 & 12 & 0 & 1 & 3 & 2 \\
\hline Home/Family & 10 & 8 & 10 & 11 & 5 & 5 & 6 & 8 \\
\hline Public/Social & 13 & 6 & 6 & 7 & 0 & 2 & 2 & 2 \\
\hline SASC-P Total Score & 63 & 57 & $55^{*}$ & $44^{*}$ & 65 & 62 & 61 & $55^{*}$ \\
\hline$F N E$ & 19 & 20 & 23 & 17 & 21 & 19 & 19 & 15 \\
\hline SAD-New & 28 & 22 & 19 & 17 & 24 & 25 & 26 & 24 \\
\hline SAD-General & 16 & 15 & 13 & 10 & 20 & 18 & 16 & 16 \\
\hline
\end{tabular}

"RCI scores of \pm 1.96 or higher indicate clinically significant changes over time.

\subsection{Social Anxiety}

For both cases, there was a noticeable decrease in level and trend in social anxiety symptoms via PAS-P ratings (see Figure 1). Effect sizes also suggest a meaningful change in PAS-P scores from baseline to midpoint ( $d=$ $1.28)$ and baseline to end-of-treatment $(d=2.69)$. Child 1 saw a clinically significant reduction in social anxiety symptoms (i.e., SASC-P) from baseline to end-of-treatment $(R C I=-2.56)$. By the three-month follow-up, reductions in social anxiety symptoms compared to baseline ratings far exceeded clinical significance for both children (Child 1: $R C I=-6.07$, Child 2: $R C I=-3.20$ ).

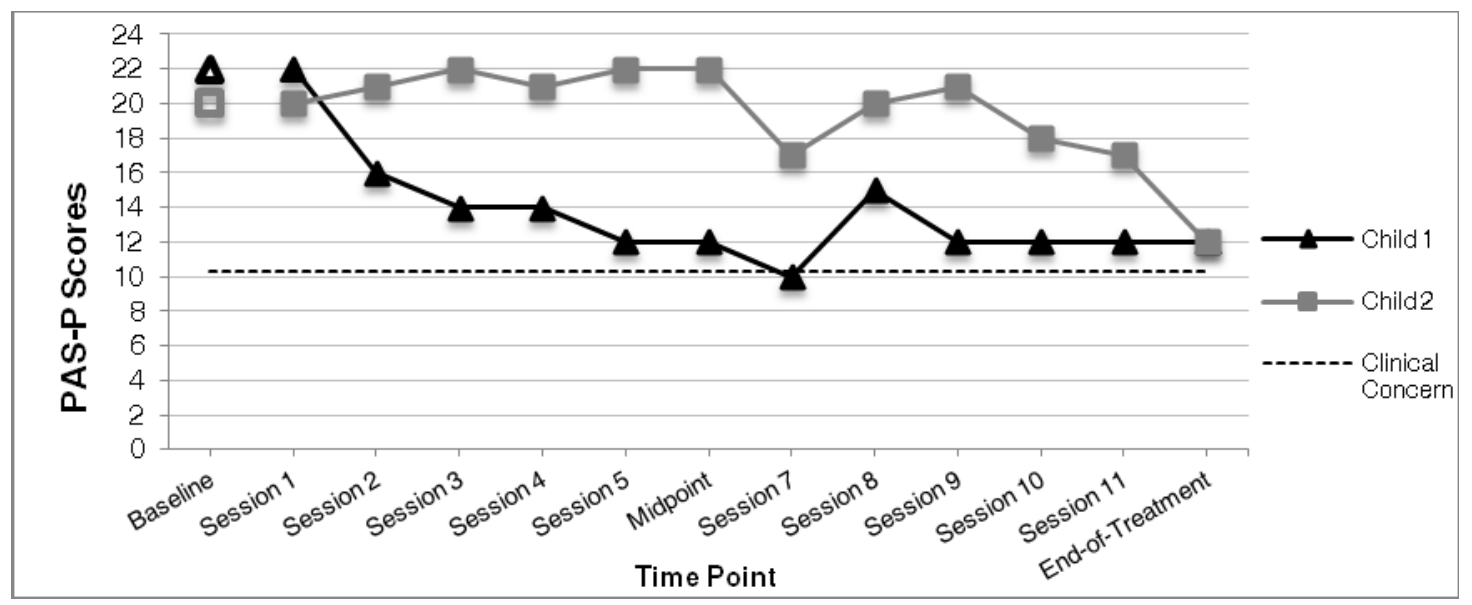

Figure 1. Weekly parent ratings of child social anxiety levels

Note. Scores on the PAS-P, Social Anxiety Subscale scores range from 0-24, with lower scores indicating low anxiety. Scores at or above one standard deviation $(S D=4.87)$ from the mean for four-year-old males $(M=5.47)$ warrant clinical investigation (score $\geq 10.34$; Spence, n.d.). 


\subsection{Treatment Acceptability}

At the end-of-treatment, parents of both children reported high overall satisfaction $(M=105.5)$ with IBTSM. Subscale scores at or above 55, 36, and 9 note high satisfaction for Acceptability, Effectiveness, and Time, respectively (Kratochwill, Elliott, Loitz, Sladeczek, \& Carlson, 2003). Parent ratings on the TEQ-P subscales exceeded these cut-offs, indicating that parents viewed IBTSM as highly acceptable $(M=61.5)$, effective $(M=$ 36.3), and appropriate for the time required $(M=11.3)$.

\section{Discussion}

This study evaluated the integrity, effectiveness, and acceptability of a condensed (12-session) version of IBTSM, a manualized approach to SM treatment carried out within a clinical setting. Overall, both cases saw some improvement in one or more of their SM symptoms after receiving 12 sessions of IBTSM over the course of 18 weeks. Observations and scores from integrity checklists clearly showed the 12-session modified IBTSM was carried out with high treatment integrity, as was seen in the original pilot study of IBTSM (Bergman et al., 2013).

Results related to diagnostic changes were mixed. Child 1, but not Child 2, saw a removal of SM diagnosis at the end of treatment. However, parents of both children reported a general increase in speech over time. Differential effects for these two cases may reflect differing severities, subtypes of SM, or the presence of comorbid disorders, as was seen in Child 1. Child 2 began treatment with a higher baseline severity and less frequent parent-reported speech. Thus, the length of treatment may have been insufficient for him to make significant gains. While Bergman and colleagues (2013) did not explore the relationship between baseline severity ratings and end-of-treatment outcomes, Oerbeck, Stein, Pripp, and Kristensen (2015) did find that children who began treatment with greater symptom severity tended to demonstrate less substantial gains.

Decreases in levels of social anxiety over the course of IBTSM treatment reached clinical significance for both cases. This reduction in social anxiety corroborates findings from the pilot study, where children showed a significant decrease in social anxiety from baseline to midpoint (session 12) and to end-of-treatment (session 20; Bergman et al., 2013). Due to our mixed findings overall, it was essential to examine acceptability data to understand parents' perceptions of IBTSM. Parents of both cases reported high acceptability with IBTSM, as was expected given high satisfaction ratings in the IBTSM pilot study (Bergman et al., 2013).

\subsection{Limitations}

Numerous limitations are associated with case study designs and generalization of results to others is not possible as these results help to identify additional questions to be addressed in future research. One important limitation of the IBTSM treatment approach was uncovered by the study psychologist. Specifically, the steps outlined in the IBTSM manual may be insufficient in guiding clinicians on how to incorporate specific behavioral strategies (e.g., shaping) to encourage different types of speech. The IBTSM manual suggests similar techniques, but included few explicit explanations on how and when to implement them. The study psychologist also noted a lack of opportunities to model and practice strategies with parents during sessions.

Finally, the study psychologist expressed a need for stronger school participation. Clearer guidance on encouraging school participation throughout the manual appears needed. While teacher involvement was mentioned early in the IBTSM manual, there was little structure in place for continually communicating with teachers. As such, school-based exposure activities were difficult to implement and data collection from teachers was unsuccessful for both cases in this study. Since school is often the context in which symptoms are more severe, active participation from teachers and frequent monitoring of progress in the school setting may be particularly essential to SM treatment (Muris \& Ollendick, 2015; Oerbeck et al., 2015).

\subsection{Implications for Research and Practice}

As the first to investigate IBTSM in an independent setting, the present study brings a few unique contributions to current literature on SM. First, there is little research examining the effectiveness of a manualized approach to SM treatment. Our results indicate IBTSM is a potentially effective manualized treatment that can be carried out when clinicians adhere to the manual. Significant replicable reductions in social anxiety suggest a need for attention to anxiety, in addition to speech avoidance, when examining effects of SM treatment. Our overall findings add to the growing literature supporting behavioral therapy as an effective approach for treating children with SM (Muris \& Ollendick, 2015; Viana, Beidel, \& Rabian, 2009).

Further research is needed to explore how school involvement affects treatment outcomes for children with SM. School-based practitioners may wish to use this manualized approach to support students with SM. More specifically, school psychologists can serve as the main point of contact for the child's therapist or may directly 
implement this manualized approach, if appropriately trained, given how important the school context appears throughout IBTSM.

\section{Acknowledgements}

This study was made possible through grant funding from the Michigan State University College of Education, the Graduate School, and the Department of Counseling, Educational Psychology, and Special Education.

\section{References}

American Psychiatric Association. (2013). Diagnostic and statistical manual of mental disorders (5th ed.). Arlington, VA: American Psychiatric Publishing. https://doi.org/10.1176/appi.books.9780890425596

Bergman, R. L. (2013). Treatment for children with selective mutism: An integrative behavioral approach. New York, NY: Oxford University Press.

Bergman, R. L., Gonzalez, A., Piacentini, J., \& Keller, M. L. (2013). Integrated Behavior Therapy for Selective Mutism: A randomized controlled pilot study. Behaviour Research and Therapy, 51, 680-689. https://doi.org/10.1016/j.brat.2013.07.003

Bergman, R. L., Keller, M. L., Piacentini, J., \& Bergman, A. J. (2008). The development and psychometric properties of the Selective Mutism Questionnaire. Journal of Clinical Child and Adolescent Psychology, 37, 456-464. https://doi.org/10.1080/15374410801955805

Jacobson, N. S., \& Truax, P. (1991). Clinical significance: A statistical approach to defining meaningful change in psychotherapy research. Journal of Consulting and Clinical Psychology, 59, 12-19. https://doi.org/10.1037/0022-006x.59.1.12

Kazdin, A. E. (1980). Acceptability of alternative treatments for deviant child behavior. Journal of Applied Behavior Analysis, 13, 259-273. https://doi.org/10.1901/jaba.1980.13-259

Kelley, M. L., Heffer, R. W., Gresham, F. M., \& Elliott, S. N. (1989). Development of a modified treatment evaluation inventory. Journal of Psychopathology and Behavioral Assessment, 11, 235-247. https://doi.org/10.1007/bf00960495

Kratochwill, T. R., Elliott, S. N., Loitz, P. A., Sladeczek, I., \& Carlson, J. S. (2003). Conjoint consultation using self-administered manual and videotape parent-teacher training: Effects on children's behavioral difficulties. School Psychology Quarterly, 18, 269-302. https://doi.org/10.1521/scpq.18.3.269.22574

La Greca, A. M., \& Stone, W. L. (1993). The social anxiety scale for children-revised: Factor structure and concurrent validity. Journal of Clinical Child Psychology, 22, 17-27. https://doi.org/10.1207/s15374424jccp2201_2

Letamendi, A. M., Chavira, D. A., Hitchcock, C. A., Roesch, S. C., Shipon-Blum, E., Stein, M. B., \& Roesch, S. C. (2008). The Selective Mutism Questionnaire: Measurement Structure and Validity. Journal of the American Academy of Child and Adolescent Psychiatry, 47, 1197-1204. https://doi.org/10.1097/CHI.0b013e3181825a7b

Muris, P., \& Ollendick, T. H. (2015). Children who are anxious in silence: A review on selective mutism, the new anxiety disorder in DSM-5. Clinical Child and Family Psychology Review, 18, 151-169. https://doi.org/10.1007/s10567-015-0181-y

Muris, P., Schmidt, H., \& Merckelbach, H. (2000). Correlations among two self-report questionnaires for measuring DSM-defined anxiety disorder symptoms in children: The Screen for Child Anxiety Related Emotional Disorders and the Spence Children's Anxiety Scale. Personality and Individual Differences, 28, 333-346. https://doi.org/10.1016/s0191-8869(99)00102-6

Oerbeck, B., Stein, M. B., Pripp, A. H., \& Kristensen, H. (2015). Selective mutism: Follow-up study 1 year after end of treatment. European Child \& Adolescent Psychiatry, 24, 757-766. https://doi.org/10.1007/s00787-014-0620-1

Silverman, W. K., \& Albano, A. M. (1996). Anxiety Disorders Interview Schedule for DSM-IV: Parent interview schedule (Vol. 1). Oxford University Press.

Silverman, W. K., Saavedra, L. M., \& Pina, A. A. (2001). Test-retest reliability of anxiety symptoms and diagnoses with the anxiety disorders interview schedule for DSM-IV: Child and parent versions. Journal of the American Academy of Child \& Adolescent Psychiatry, 40, 937-944. https://doi.org/10.1097/00004583-200108000-00016 
Spence, S. H. (n.d.). Preschool Scale: Interpretation of Scores. Retrieved from http://www.scaswebsite.com/index.php?p=1_30

Spence, S. H., Rapee, R., McDonald, C., \& Ingram, M. (2001). The structure of anxiety symptoms among

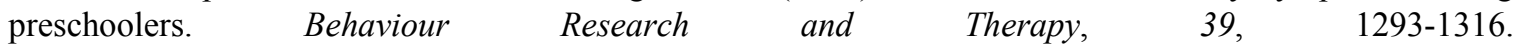
https://doi.org/10.1016/s0005-7967(00)00098-x

Steinhausen, H., Wachter, M., Laimböck, K., \& Metzke, C. W. (2006). A long-term outcome study of selective mutism in childhood. Journal of Child Psychology and Psychiatry, 47, 751-756. https://doi.org/10.1111/j.1469-7610.2005.01560.x

Viana, A. G., Beidel, D. C., \& Rabian, B. (2009). Selective mutism: A review and integration of the last 15 years. Clinical Psychology Review, 29, 57-67. https://doi.org/10.1016/j.cpr.2008.09.009

\section{Copyrights}

Copyright for this article is retained by the author(s), with first publication rights granted to the journal.

This is an open-access article distributed under the terms and conditions of the Creative Commons Attribution license (http://creativecommons.org/licenses/by/4.0/). 